\title{
Snipping away at the HIV pandemic, one foreskin at a time
}

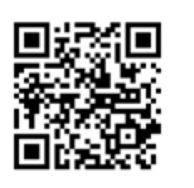

Nearly two million South African (SA) men will have been voluntarily circumcised by the end of this year, about half of the government's original target - yet even this will have conservatively prevented 133333 new HIV infections as a timely new public/private sector initiative kicks in to boost overall efforts. In spite of this, alarm bells continue to ring as interventions for women, twice as vulnerable biologically and culturally, remain in various stages of trial.

Proven in field trials to reduce the risk of HIV infection by $60 \%$ and calculated to prevent one HIV infection for every 5 - 15 men circumcised, voluntary medical male circumcision (VMMC) is uniquely suited to the SA environment, where 1400 new HIV infections occur daily, the vast majority via heterosexual transmission. The latest private sector intervention comes from Metropolitan Health Risk Management, who have partnered with the Centre for HIV/AIDS Prevention Studies (CHAPS) and the National Department of Health in a bid to halve new HIV infections by next year - via VMMC scale-up. The ZAR55 million United States Agency for International Development (USAID)-funded initiative is two-pronged: training or upskilling 850 general practitioners, and increasing VMMC coverage in the private sector to 100000 men per year by 2017 . Since 2007 , what was originally a Metropolitan Health and CHAPS initiative (government endorsed the programme in April this year) has resulted in 170000 Metropolitan Health members being voluntarily circumcised. The private sector initiative uptake has slowly accelerated, from 12000 in 2008 to 20000 in 2010, through 30000 in 2012 to 45000 last year. Mr Siraaj Adams, General Manager for HIV and TB at Metropolitan Health, said that the ZAR55 million was going towards the voluntary training of GPs by CHAPS at government facilities after their practices were identified according to HIV prevalence demographics (Johannesburg, Pretoria, Nelspruit, Uitenhage and Cape Town so far). By early June this year, a total of $150 \mathrm{GPs}$ had undergone the rigorous training and been set up at their practices, where they were provided with free surgical circumcision packs and HIV test kits, and paid either the going medical aid rates for insured patients or a median medical aid rate by USAID for patients without cover. Doctors had to be covered for adverse events through medical practice insurance and earned ten continuing professional development (CPD) points after being 'signed off' as competent VMMC/CHAPS practitioners. Adams said that the theoretical and practical course (plus the in-practice 'sign-off' review) was oversubscribed, with 20 doctors on the waiting list for the next two training weekends (at the time of writing). Up to eight doctors were trained in one government facility at a time. The training and support service incorporates a comprehensive package of services, including HIV testing, sexually transmitted infection (STI) management, VMMC and condom provision. No sexual contact for 6 weeks after the procedure and condom use are strongly encouraged (circumcision often being perceived as an 'invisible condom').

\section{Funder exclusion}

\section{barriers removed}

A significant development in the lead-up to the current public/private partnership was that all medical aid schemes (both open and closed) in the Metropolitan stable removed all exclusions around VMMC (in 2012). Before that, members who applied on non-medical grounds were automatically excluded - a significant barrier to HIV prevention efforts. The offer of no outof-pocket costs for VMMC services by GPs will vastly improve accessibility, with Metropolitan Health data suggesting that $35 \%$ of VMMCs were already being done by GPs, up to $65 \%$ of circumcisions being conducted as day 
procedures in hospital. To give some idea of the patient savings of the new initiative, a private GP visit for the procedure costs around ZAR1 000 while a private in-hospital procedure could cost well into double figures. Over a 10 -year time horizon, it is estimated that VMMC in highprevalence areas will save between ZAR1 650 and ZAR9 900 per infection prevented. Adams said that in Gauteng alone, if 1000 adult males were circumcised, ZAR26.4 million could be saved in HIV treatment over 20 years. This comprehensively negated arguments that the money for VMMC budgets could be better used in other HIV prevention strategies. 'The very opposite is true: VMMC frees up money for other strategies to be enhanced', he said. Metropolitan Health schemes include several closed government schemes such as Polmed, Transmed and GEMS, making it the largest administrator in the country with three million lives covered (followed by Discovery and Medscheme). Adams revealed that HIV prevalence among Metropolitan Health members stood at $6.5 \%$. He appealed to other medical schemes to also scrap VMMC exclusions on non-medical grounds, saying that the benefits of the new project accrued to members of any medical scheme and that they were a good example of how the private and public sectors could work together for the benefit of all in the impending National Health Insurance.

\section{Government response}

Current government VMMC efforts began 3 years ago after HIV prevention efficacy became clear from the original Orange Farm pilot study in Gauteng and two other sub-Saharan sites. It was initiated after comprehensive consultation with all 19 stakeholders in the South African National AIDS Council and discussion on scientific, social and cultural issues. It is now official policy and part of the 2012 - 2016 National Strategic Plan for HIV, STIs and TB. VMMC is regarded as a 'game-changer' in SA's HIV prevention efforts, and all provinces are prioritising efforts to accelerate access.

Asked about the possible inclusion of the Prepex, a non-surgical three-ringed device that results in the foreskin atrophying and falling off after about seven days and is currently being piloted by government in four provinces, Adams said it was being excluded until officially 'signed off'. While the safety and efficacy of the device were very high, 'we are promoting the surgical technique (forceps guided and dorsal slit) the intention is that non-surgical techniques will become an option'. Prepex's greatest contribution to the HIV prevention battle is that is relatively pain-free, and can be

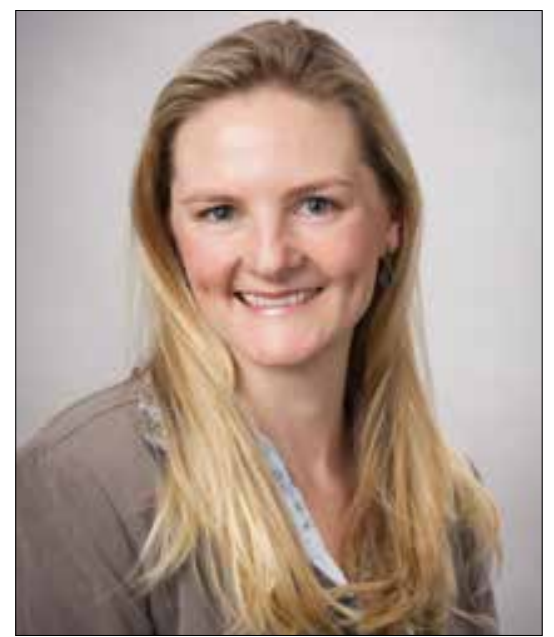

CAPRISA research associate Dr Cheryl Baxter.

prescribed and fitted by a nurse, enabling task-shifting down from over-burdened GPs. A downside is that a Prepex wound takes 8 weeks to heal, 2 weeks longer than healing after surgical circumcision.

Modelling studies suggest striking implications of scaling up VMMC in averting millions of infections and deaths and saving billions of rands in the long run. Adams said any further delays would constitute a 'major failure to capitalise on scientific evidence to save lives and improve the quality of our population'.

\section{A 'jolly good show' by (and for) CHAPS}

CHAPS, a USAID-funded NGO, is a global leader in implementing and disseminating evidence-based approaches to prevent the spread of HIV in southern Africa such as efficiency models and professional training. It was behind the original sub-Saharan studies that proved the efficacy of VMMC. The main pillars of the new 3-year public-private partnership will include Metropolitan Health initially covering the costs of high-quality VMMC to over 32000 men, USAID supplying disposable circumcision packs to GPs where needed, and CHAPS assisting GPs to set up their facilities and address emergencies and adverse events, and supporting their accurate recording and reporting of VMMC services. USAID and CHAPS have also undertaken to help create demand and raise awareness of GP-provided VMMC services.

Meanwhile Dr Cheryl Baxter, an associate researcher at the Centre for the AIDS Program of Research in South Africa (CAPRISA) in Durban, said there was a paucity of any comparable data for HIV prevention in women. The only currently available HIV prevention options for women were the $\mathrm{ABCs}$ of Abstinence, Be faithful and Condomise.

\section{Women remain twice as vulnerable}

Prevention options for women, such as microbicides, long-acting vaginal rings and long-acting injectables, while holding promise, were not yet available. HIV treatment of an infected partner would have an impact on HIV prevention for women, but it was not something that a woman could control the male partner would have to know his HIV status, and if HIV-positive would need to be on antiretrovirals (and take them as prescribed) for the woman to benefit. Oral pre-exposure prophylactics had been shown to work in a number of populations (men who have sex with men, discordant couples and heterosexuals), but again this was not a currently available HIV prevention option in SA. 'There might be some data on condom scale-up, but most of the condoms distributed are male condoms,' Baxter added.

\section{'If we pursue a downward trend in financing AIDS, we see a devastating slide backward by 2020 - an increase in new HIV infections and AIDS-related deaths and escalating costs of controlling the epidemic.' - Moses-Burton}

Women bear a disproportionate burden of the HIV epidemic in sub-Saharan Africa (SSA), and account for approximately $60 \%$ of all infections in this region. HIV-infected women between the ages of 15 and 24 years represent $76 \%$ of total cases in that age group. The rapid spread of HIV among adolescent girls and young women in SA has been described as 'explosive'. A survey conducted among high-school students in one KwaZuluNatal health district showed that the HIV prevalence in girls was six times higher than in boys. National, annual, anonymous seroprevalence surveys in pregnant women using public sector healthcare facilities demonstrate that HIV prevalence in SSA has increased from $0.8 \%$ in 1990 to $30.2 \%$ in 2010. Several factors contribute to the increased vulnerability of young women to acquiring HIV in SSA, among them the fact that biologically, women appear to be more susceptible to acquiring HIV than men. According to the US Centers for Disease Control and Prevention, the risk of HIV infection is $1 / 2000$ contacts for the male partner compared with $1 / 1000$ contacts for the female partner in penile-vaginal sex. Women are twice as likely to become infected as men after a single sexual encounter. ${ }^{[1]}$ 


\section{Downscaling funding 'asking for trouble'}

The Global Network of People Living with HIV $(\mathrm{GNP}+)$ last month applauded the conclusions of the recent UNAIDS-Lancet Commission report, 'Defeating AIDS - advancing global health', i.e. that failing to continue funding the AIDS response sufficiently would have grave andimmediate consequences'. The Commission was tasked with exploring strategies to ensure that the vision of the global AIDS movement, 'Zero new HIV infections, zero discrimination and zero AIDS-related deaths', could be realised in coming decades. The report stresses that a human rights and community mobilisation approach has been central to the successes of the AIDS response to date, emphasising that failure to fund the AIDS response sufficiently, particularly the contribution of civil society will have major consequences for continued success and sustainability under the sustainable development goals.

Suzette Moses-Burton, GNP+ executive director, said the report presented two 'stark and contrasting pictures. If we pursue a downward trend in financing AIDS, we see a devastating slide backward by 2020 - an increase in new HIV infections and AIDS-related deaths and escalating costs of controlling the epidemic. In the alternative picture, with continued investment in AIDS over the next 5 years, we see HIV transmission at low endemic levels, AIDS-related mortality significantly reduced and children born HIV free'

Impressively, the UNAIDS-Lancet Commission report makes a strong economic argu- ment for ambitious investment, showing that robust financial investment in the HIV response now will create significant returns later. Each life-year gained in low- and middle-income countries produces significant gains in GDP. Moses-Burton added: 'With over 10 million people needing antiretroviral drugs today, now is not the time to backtrack on funding and political commitment. We cannot end the AIDS epidemic without that one last push.'

\section{Chris Bateman}

chrisb@hmpg.co.za

S Afr Med J 2015;105(8):623-625. DOI:10.7196/SAMJnew.8123

1. Abdool Karim S, Baxter C, Frohlich J, Abdool Karim Q. The need for multipurpose prevention technologies in sub-Saharan Africa. BJOG 2014;121(Suppl 5):27-34. [http://dx.doi.org/10.1111/1471 0528.12842] 